\title{
The bile acid composition analysis of the lipophilic complex of animal bile
}

\author{
Aleksandr Stremoukhov ${ }^{2}$, Irina Zhirova ${ }^{1 *}$, Anastasiya Goncharova $^{1}$, Yulia Korj $^{2}$, Galina \\ Varenykh $^{1}$, and Marina Shulgina ${ }^{1}$ \\ ${ }^{1}$ Belgorod State National Research University, 308015, Belgorod, Russia \\ ${ }^{2}$ National University of Pharmacy, 61002, Kharkiv, Ukraine
}

\begin{abstract}
Using gas chromatography, the qualitative composition and the quantitative content of bile acids in the lipophilic complexes of animal bile was studied. As a result of the studies, 6 steroids were identified in the lipophilic complexes of bile, 5 of which are bile acids. It was defined that cholic and deoxycholic acid predominate in all the studied objects, while their amount depends on the used raw materials.
\end{abstract}

Keywords: bile acids, lipophilic complex, glycoprotein complex, water residue of bile

\section{Introduction}

Due to the intensive development and improvement of chromatographic research methods, it became possible the detail study of individual bile acids metabolism in animals and humans, poison updating in the general chain of steroid compounds conversion, further deepen understanding of the physiological action mechanisms in the body of a number of bile acids that differ in their physico-chemical properties and structure [1,2].

According to the chemical composition, bile acids are derivatives of cholanic acid and differ only in the presence of one, two or three $\mathrm{OH}$-groups in different positions in the cyclic part of the molecule, therefore, mono -, di- and trihydroxycholanic acids are distinguished. There are also keto-alkaline acids. Free bile acids in human bile either do not appear at all, or present in very small amounts [1,3]. Primary bile acids - trihydroxycholanic (cholic) and dihydroxycholanic (henodeoxycholic) - are formed in the liver, and then by an amide bond combine with aminoacetic acid (glycine) and a sulfur-containing amine (taurine), forming so-called bile acid salts, or paired bile acids.

Physiological activity in the digestive tract is associated not with the bile acids themselves, which have low solubility in water and low surface activity, but with their conjugates and sodium and potassium salts, which are anionic detergents [10]. They promote the absorption of fats, affect intestinal peristalsis, activate enzyme systems, exhibit antiseptic properties, support and stabilize bile lipid substances, especially cholesterol, in a colloidal solution [1].

Different vertebrata species characterize with very significant differences in the composition of bile acids, that primarily indicate the presence of specific metabolism features of these compounds. Currently, more than 50 natural bile acids and their various derivatives

\footnotetext{
*Corresponding author: zhirova@bsu.edu.ru
} 
are known $[8,7]$. The search for new complexes that include bile acids and their research is relevant issue.

\section{The purpose of the study}

The aim of the study was to study the bile acid composition of lipophilic complexes (LC). The objects of the study were bile samples obtained from bile of cattle (BBt) and pigs (BSs).

\section{Materials and methods}

Determination of bile $[4,5,8,9,11]$ acids in the LC of animal bile (Bos taurus L., Sus scrofa domesticus L.) were carried out by the method of gas-liquid chromatography (GLC) on polar stationary phases with previous methylation. The allocating and registration of bile acids was carried out on a gas chromatograph "Chrome-5" on a metal column $2.6 \mathrm{~m}$ long, filled with a sorbent "Chromaton-super" with $5 \% \mathrm{oV}-17$. The analysis of samples of free bile acids was carried out in an isothermal mode at $200{ }^{\circ} \mathrm{C}$ and heating of the flame-ionization detector $250{ }^{\circ} \mathrm{C}$. The speed of the carrier gas of high purity nitrogen is $50 \mathrm{ml} / \mathrm{min}$, hydrogen - 30 $\mathrm{ml} / \mathrm{min}$, air - $300 \mathrm{ml} / \mathrm{min}$.

A $300 \mathrm{mg}$ bile sample was placed in a $25 \mathrm{ml}$ flask, $6 \mathrm{ml}$ of Folch solution (chloroform methanol 2:1) was added for the dissolution of lipophilic components of bile and separation from protein in the dry fraction of bile. The contents were mixed and heated on a water heater at $50{ }^{\circ} \mathrm{C}$ for 5 minutes. Samples with a dry fraction were centrifuged at 3 thousand rpm for 10 minutes. $10 \mathrm{ml}$ of $20 \% \mathrm{NaOH}$ solution ware added to the solutions and hydrolysis was carried out at $150{ }^{\circ} \mathrm{C}$ for 30 minutes. The test tubes were cooled, $5 \mathrm{ml}$ of ethyl acetate was added, thoroughly mixed, and after retention, the upper phase was sampled, transferred to a $40 \mathrm{ml}$ reaction tube, evaporated to a dry residue on an water heater under nitrogen. $0.1 \mathrm{ml}$ of trimethyltrichloroselene solution was added to the dry residue in order to convert it into an inert compound. To replace the active groups of bile acids, the sample was subjected to additional methylation with $5 \%$ sulfuric acid in methanol at $80{ }^{\circ} \mathrm{C}$ for 30 minutes. After cooling the samples, $3 \mathrm{ml}$ of purified water was added, intensively shaken, $5 \mathrm{ml}$ of a mixture of chloroform-hexane-methanol was added in a ratio of 10: 10:1, thoroughly mixed and after retention, the upper phase was selected, transferred to a centrofuge tube, in which the solution was evaporated to $0.5-2 \mathrm{ml}$, for insertion into a gas chromatograph. Qualitative analysis was carried out by comparing the retention time of bile acids with known samples. The quantitative content is calculated by the formula:

$$
C_{x}=\frac{S_{\text {samhel }} \cdot C_{s t}}{S_{s t}}
$$

where:

$\mathrm{C}_{x}$ is the concentration of bile acid in the complex, $\mathrm{mg}$;

$\mathrm{C}_{s t}$ is the concentration of standard bile acid, $\mathrm{mg}$;

$\mathrm{S}_{\text {sample }}$ is the area of the peak of bile acid in the complex, sq.mm;

$\mathrm{S}_{s t}$ is the peak area of standard bile acid, sq.mm.

\section{Results and discussion}

The results of the study of the steroid composition of animal bile and LC bile are shown in Tables 1-2 and Fig. 1. 
Table 1. The content of steroids in native bile

\begin{tabular}{|c|c|c|c|c|}
\hline \multirow{2}{*}{ Name } & \multicolumn{2}{|c|}{ BBt } & \multicolumn{2}{c|}{ BSs } \\
\cline { 2 - 5 } & $\mathbf{m m o l} / \mathbf{L}$ & $\mathbf{~ m g} / \mathbf{m l}$ & $\mathbf{~ m m o l} / \mathbf{L}$ & $\mathbf{m g} / \mathbf{m l}$ \\
\hline Total cholesterol & $4,89 \pm 0,15$ & $1,56 \pm 0,08$ & $6,12 \pm 0,18$ & $2,33 \pm 0,12$ \\
\hline Bile acids & $23,13 \pm 0,70$ & $9,50 \pm 0,50$ & $31,40 \pm 0,95$ & $12,89 \pm 0,68$ \\
\hline
\end{tabular}

The content of bile acids in the BSs is 1.36 times higher than in the BBt and cholesterol is 1.49 times higher, which indicates the prospects of using BSs to produce bile acids.

For bile LC, methylation was carried out without adding a Folch solution and centrifugation, and began with the addition of $5 \mathrm{ml}$ of ethyl acetate and $10 \mathrm{ml}$ of $20 \% \mathrm{NaOH}$ solution, then followed the procedure described above. The results of the analysis are presented in Table 2.

Table 2. The concentration of bile acids and cholesterol in the bile LC

\begin{tabular}{|c|c|c|c|c|}
\hline \multirow{2}{*}{ Name } & \multicolumn{2}{|c|}{$\mathbf{B B t}$} & \multicolumn{2}{c|}{ BSs } \\
\cline { 2 - 5 } & $\mathbf{m m o l} / \mathbf{L}$ & $\mathbf{m g} / \mathbf{m l}$ & $\mathbf{m m o l} / \mathbf{L}$ & $\mathbf{~ m g / m l}$ \\
\hline 1 & 2 & 3 & 4 & 5 \\
\hline Cholesterol & $2,55 \pm 0,13$ & $0,99 \pm 0,05$ & $3,19 \pm 0,17$ & $1,23 \pm 0,06$ \\
\hline Cholic acid & $47,31 \pm 2,50$ & $19,71 \pm 1,04$ & $35,48 \pm 1,87$ & $14,79 \pm 0,78$ \\
\hline Ursolic acid & $3,10 \pm 0,16$ & $1,29 \pm 0,07$ & $2,98 \pm 0,16$ & $1,24 \pm 0,07$ \\
\hline Deoxycholic acid & $43,76 \pm 2,31$ & $18,24 \pm 0,96$ & $37,85 \pm 2,00$ & $15,77 \pm 0,83$ \\
\hline Henodeoxycholic acid & $28,39 \pm 1,50$ & $11,83 \pm 0,62$ & $26,02 \pm 1,37$ & $10,84 \pm 0,57$ \\
\hline Litocholic acid & $3,55 \pm 0,19$ & $1,48 \pm 0,08$ & $0,71 \pm 0,04$ & $0,30 \pm 0,02$ \\
\hline Total bile acids & $126,11 \pm 6,65$ & $52,55 \pm 2,77$ & $103,05 \pm 5,44$ & $42,94 \pm 2,26$ \\
\hline
\end{tabular}

The quantitative determination showed that the highest content of bile acids is in the LC of BBt - 1.22 times more than in the LC of BSs, while the cholesterol content in the LC of BBt is 1.24 times less than in the LC of BSs. As can be seen from Table 2, as a result of the study, 6 steroid compounds were identified in the bile LC, of which 5 belong to bile acids. It was found that cholic and deoxycholic acid predominate in all the studied objects, while there is more cholic acid in LC BBt and more deoxycholic acid in LC BSs.

Comparing the content of cholesterol and bile acids in native bile (Table. 1) and the obtained LC (Table. 2), it was found that the concentration of bile acids increases about 5.45 times for BBt and 3.28 times for BSs, and cholesterol decreases by 1.9 times. The results of the comparative analysis are presented in the diagram (Fig. 1).

The technology for obtaining LC bile also makes it possible to obtain a glycoprotein complex (GPC) and a water residue of bile (WRB), for the creation on their basis of new drugs with established pharmacological activity.

By methods of liquid-liquid extraction and chromatography in the complexes of animals bile the presence of 137 compounds was established: bile acids (45), fatty acids (20), amino acids (17), among them - 9 essential, 14 fatty acids, phospholipids (13), cholesterol (1), porphyrins (16), enzymes (6), macro-and micronutrients (19). By two-dimensional chromatography in the LC of bile revealed 75 substances which by chromatographic mobility, fluorescence under UV-light and the colouring with specific reagents were referred to steroids (46), phospholipids (13), porphyrins (16). 


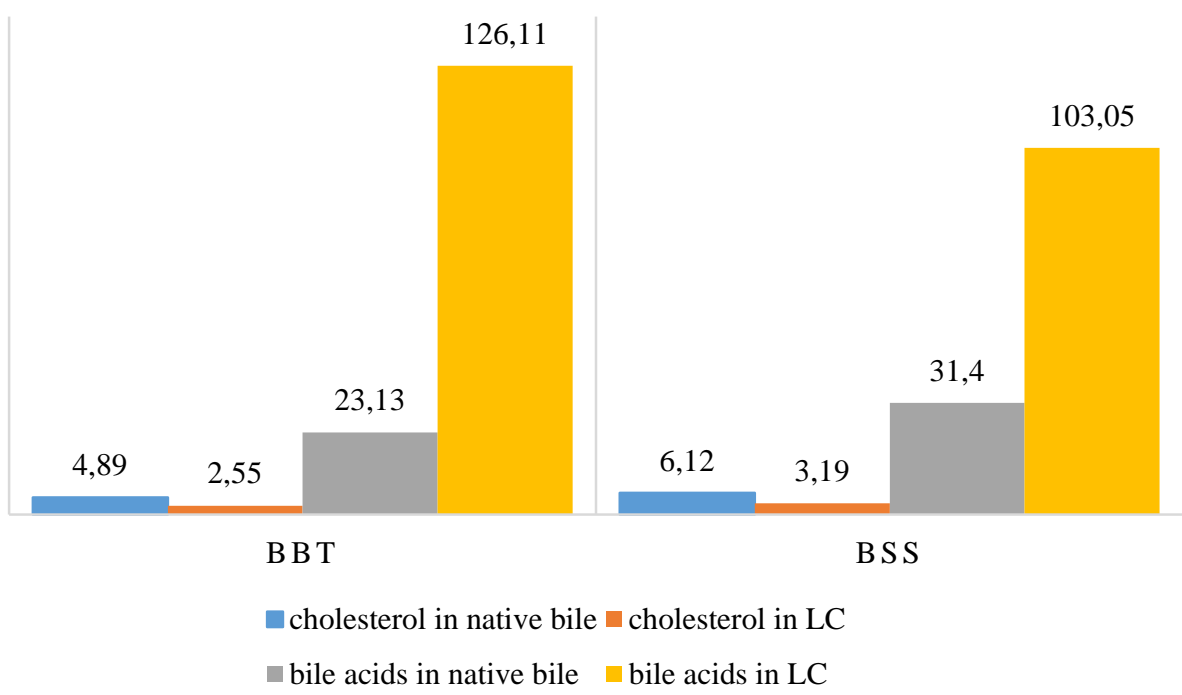

Fig. 1. Comparative content of steroids in native bile and LC (mmol/L)

Chinese medicine has incorporated the use of bear gall bladders and bile for over 1300 years. The Pharmacopoeia of the People's Republic of China (1977) indicates that bear gall bladder is useful for cleansing heat symptoms, calming the liver and brightening the eyes. It is also used for treating convulsions, conjunctivitis and pharyngolaryngitis. Pharmacological and clinical studies have confirmed the therapeutic effects of bear bile, especially of its components, ursodeoxycholic acid (UDCA) and chenodeoxycholic acids (CDCA), for the treatment of gallstones and liver diseases. Pig bile has also been found to have antiinflammatory, nticonvulsant and analgesic effects, and can prolong the survival time of mice under hypoxic conditions. The bile of mammals, as well as of other animal species, results from metabolic processes in the liver. Bile consists of approximately $86 \%$ water and $14 \%$ dry matter. The most important components of the dry matter are free and conjugated bile acids.

The antimicrobial activity of LC in concentration 0,1\% against Staphylococcus aureus, Pseudomonas aeruginosa, Bacillus subtilis and Candida albicans and antibacterial activity of WRB BBt towards Staphylococcus aureus and Candida albicans were established. Pharmacological researches were conducted in vivo and in vitro. It was established that bile GPC helps absorption of fats, activate lipase in digestion and stimulates peristalsis in experimental animals.

The WRB has expressed antihypoxic action and stabilizes the membranes. LC showes pronounced hepatotropic and antioxidant effects. WRB in a dose of $12 \mathrm{ml} / \mathrm{kg}, \mathrm{LC}-20 \mathrm{mg} / \mathrm{kg}$, $\mathrm{GPC}-25 \mathrm{ml} / \mathrm{kg}$ at 30-day intragastric introduction do not have toxic effects on the peripheral blood picture and morpho-functional state of the internal organs in the subacute experiment. Complexes can be recommended as medications to improve digestion, in violation of cerebral and peripheral circulation, redox metabolism in tissues, at hepatitis, as a tonic, stabilizing agent in adverse effects.

\section{Conclusions}

1. The qualitative composition was studied by gas chromatography and the quantitative content of bile acids and cholesterol in native bile and LC was established. 
2. The conducted studies have shown a high content of bile acids in the bile LC, which allows to recommend these types of raw materials and complexes for the production of drugs with hepatoprotective and antioxidant activity.

\section{References}

1. Y.V. Ganitkevich, Successes of physiological sciences, 15, 4 (1984)

2. L.V. Molostova, Biol. S., 5 (1987)

3. S.S. Alil, H. Farhat, W.H. Elliott, J. Lipid Res, 17, 21-24 (1976)

4. A.K. Batta, G.Salen, J. Chromatog. B, 723, 1-16 (1999)

5. M.L. Jones, H. Chen, W. Ouyang, J. Med. Sci, 23, 277-280 (2003)

6. G. Kakiyama, T. Iida, T. Goto, J. Lipid Res, 47, 1551-1558 (2006)

7. Mikov M. Bile acids: Chemistry, Biosynthesis, Analysis, Chemical and Metabolic Transformations and Pharmacology: Monograph / Momir Mikov, J. Paul Fawcett, Ksenija Kuhajda et al. - Geneva: Mediset-publishers (2007)

8. S.S. Rossi, J.L. Converse, A.F. Hofmann, J. Lipid Res, 28, 589-595 (1987)

9. I. E. Sambrook, J. Sci. Food Agric., 32, 781-791 (1981)

10. C. Sarbu, K. Kuhajda, S. Kevresan, J. Chromatogr. A., 917, 361-366 (2001)

11. Y. H. Yeh, D. F. Hwang, J. Chromatogr. B., 751, 1-8 (2001) 